\title{
Global distribution of the thermospheric disturbances produced by effects from the upper and lower regions: simulations by a whole atmosphere GCM
}

\author{
Hitoshi Fujiwara $^{1}$ and Yasunobu Miyoshi ${ }^{2}$ \\ ${ }^{1}$ Department of Geophysics, Graduate School of Science, Tohoku University, Sendai, Japan \\ ${ }^{2}$ Department of Earth and Planetary Science, Faculty of Sciences, Kyushu University, Fukuoka, Japan \\ (Received September 30, 2007; Revised February 10, 2008; Accepted May 30, 2008; Online published May 14, 2009)
}

\begin{abstract}
It is well-known that low-latitude ionospheric/thermospheric disturbances are sometimes generated in association with the passage of traveling ionospheric/atmospheric disturbances (TIDs/TADs) produced in the highlatitude region and that the low-latitude ionosphere/thermosphere should be strongly coupled with the lower atmosphere. These facts suggest that the appearance of thermospheric disturbances with complex structures in the low-latitude region are the result of a superposition of disturbances which have different origins. We have investigated the lower atmospheric effects on the morphology of the thermospheric disturbances in response to changes in the geomagnetic activity by using a whole atmosphere general circulation model (GCM). In order to suppress the lower atmospheric effects, we set the global mean temperature and zero-wind below about 80-km altitude in the GCM. The simulation results show that the lower atmospheric effects can produce latitudinal and longitudinal structures in the low-latitude thermosphere. These lower atmospheric effects also modulate the amplitudes and structures of TADs propagating from the high- to low-latitude regions. Our results suggest that the lower atmospheric effects can produce variability in the TIDs/TADs, which in turn would create conditions conducive to plasma instabilities in the low-latitude ionosphere.
\end{abstract}

Key words: Thermosphere, GCM, simulation, traveling atmospheric disturbances (TADs), atmospheric coupling.

\section{Introduction}

Most of the fundamental features of the thermosphere/ionosphere, such as large-scale circulation, composition structure, and global mean energy budget, are well understood owing to previous observational and modeling efforts. In contrast, the sources, generation mechanisms, and characteristics of localized, small-scale, and timevarying thermospheric/ionospheric disturbances are not yet well understood.

A number of recent observations and numerical simulations have suggested the presence of lower atmospheric effects on the $F$-region ionosphere/thermosphere (e.g., Mendillo et al., 2002; Miyoshi and Fujiwara, 2003; Takahashi et al., 2005; Balan et al., 2006; Immel et al., 2006; Lühr et al., 2007). For example, Immel et al. (2006) showed a four-wave structure in the equatorial ionospheric anomaly (EIA) from the IMAGE satellite observations. These authors suggested that tidal waves modulate the $E$-region ionospheric dynamo to affect the $F$-region ionosphere. Lühr et al. (2007) also detected persistent and dominant four-peaked longitudinal variations in the EIA and zonal delta-wind (deviation from zonal average) from CHAMP satellite observations. They interpreted these structures as being caused by the eastward propagating diurnal tide with

Copyright (c) The Society of Geomagnetism and Earth, Planetary and Space Sciences (SGEPSS); The Seismological Society of Japan; The Volcanological Society of Japan; The Geodetic Society of Japan; The Japanese Society for Planetary Sciences; TERRAPUB. a wavenumber of three excited by latent heat release in the tropical troposphere.

In addition to the above lower atmospheric effects, it is well-known that low-latitude ionospheric/thermospheric disturbances are sometimes generated in association with the passage of traveling ionospheric/atmospheric disturbances (TIDs/TADs) which are caused by high-latitude energy inputs from the magnetosphere (e.g., Millward et al., 1993; Fujiwara et al., 1996; Balthazor and Moffett, 1999; Bruinsma et al., 2006; Fujiwara and Miyoshi, 2006). Recent simulations by Fujiwara and Miyoshi (2006) showed the generations and propagations of large-scale TADs in both cases of geomagnetically disturbed and quiet periods. They also suggested the importance of coupling between the lower and upper atmospheric effects for producing disturbances in the upper thermosphere. In addition, as pointed out by Nicolls and Kelley (2005), interactions between TIDs/TADs and atmospheric waves originating in the lower atmosphere should be important to the creation of suitable conditions for the generation of plasma instabilities.

In order to understand the thermospheric dynamics and energetics which vary from hour to hour, thermospheric disturbances produced by both effects from the lower atmosphere and the magnetosphere should be investigated. We have focused our attention on lower atmospheric effects on the production of thermospheric localized disturbances and the superposition of disturbances which have different ori- 
gins. The characteristics of such disturbances have not yet been clarified.

Miyoshi and Fujiwara (2003) developed a general circulation model (GCM) which covered the altitude regions from the ground to the top of the thermosphere (or exobase). This whole atmosphere GCM enables us to investigate variations of thermospheric dynamics and energetics caused by atmospheric waves propagating from the lower atmosphere and the high-latitude energy and momentum inputs from the magnetosphere (Miyoshi and Fujiwara, 2003, 2006; Fujiwara and Miyoshi, 2006). Using the GCM, we performed the numerical simulations described in the following section with the aim of clarifying the morphological characteristics of disturbances appearing in the upper thermosphere. Note that in our study the word "localized disturbances" is sometimes used to refer to disturbances with smaller spatial scales than the day-night distribution.

\section{GCM and Simulations}

The GCM used in this study has been developed as an extension of the middle atmosphere GCM developed at Kyushu University (Miyahara et al., 1993; Miyoshi, 1999). The present GCM is a global spectral model with a triangular truncation of T21 (the maximum horizontal wave number is equal to 21 , with spatial scale of about $5.6^{\circ}$ in latitude and longitude). The GCM includes 75 vertical levels and covers the regions from the ground surface to the exobase (which has a height of about $500 \mathrm{~km}$ for the solar minimum and geomagnetically quiet condition) with a vertical resolution of 0.4 scale height above the tropopause. The GCM has a full set of the physical processes appropriate for the troposphere, stratosphere, mesosphere, and thermosphere. Since the GCM has already been described in detail in previous reports (Miyoshi and Fujiwara, 2003, 2006; Fujiwara and Miyoshi, 2006; and references therein), we provide here only a brief description of the physical processes in the upper atmosphere.

In the thermosphere, the major species are assumed to be $\mathrm{N}_{2}, \mathrm{O}_{2}$, and $\mathrm{O}$. The continuity equation of mass mixing ratio for the major species is solved. The time step for integrating the equations is $100 \mathrm{~s}$. The displacement of the geomagnetic poles relative to the geodetic poles is considered in the GCM. The atmospheric heating due to absorption of the solar extreme ultraviolet (EUV) and ultraviolet (UV) radiations is considered. In addition, the magnetospheric convection electric field modeled by Volland (1975) and empirical ionosphere based on Chiu (1975), Fuller-Rowell and Evans (1987), and Roble and Ridley (1987) are used to calculate the Joule heating rate, auroral particle heating rate, and ion-drag force. The infrared cooling processes due to radiations from $\mathrm{CO}_{2}, \mathrm{NO}$, and $\mathrm{O}$ are also considered in the GCM.

In order to compare our present results with our previous TAD simulations, we assumed the same condition to the simulations as Fujiwara and Miyoshi (2006); the solar minimum condition $\left(F_{10.7}=70\right)$ is assumed, and the starttime of simulations is at 00:00 UT on November 5. We performed numerical simulations in the cases of geomagnetic quiet (cross polar cap potential drop, $\Phi$, is $30 \mathrm{kV}$ ) and disturbed (during the first $1 \mathrm{~h}, \Phi$ is enhanced to $60 \mathrm{kV}$ and is set to $30 \mathrm{kV}$ again) periods, with and without the lower atmospheric effects. We set the temperature to be global mean values (namely, horizontally uniform) calculated by the GCM, and winds are set to be zero below an altitude of about $80 \mathrm{~km}$ when the lower atmospheric effects are excluded from the upper atmosphere.

\section{Results}

We first show the fundamental feature of the thermosphere described by the whole atmosphere GCM. A global mean temperature profile for all altitude regions at 00:00 UT on November 5 is shown in Fig. 1. A solar minimum and geomagnetically quiet condition is assumed. The temperature and height at the exobase are $767 \mathrm{~K}$ and $501 \mathrm{~km}$, respectively; those at the mesopause are $178 \mathrm{~K}$ and $95 \mathrm{~km}$, respectively.

Figure 2(a-f) shows horizontal distributions of temperature and horizontal wind pattern on the constant pressure surfaces of about 90 (a), 96 (b), 108 (c), 112 (d), 116 (e), and 122 (f) km (global mean altitudes) at 00:00 UT on November 5 . The ordinate and abscissa are geographic latitude and longitude, respectively, in each panel. Note that the temperature range is different in each panel, with the maximum wind velocity in each panel of Fig. 2 being 190 (a), 182 (b), 175 (c), 181 (d), 164 (e), and 144 (f) m/s. Hightemperature regions are seen at low latitudes, with localized structures in all the panels of Fig. 2, suggesting that the results are due to the effects of upward propagating and in-situ generated atmospheric waves/disturbances. At altitudes of about 116 and $122 \mathrm{~km}$ (Fig. 2(e, f)), high-temperature regions appear at high latitudes in the southern hemisphere (almost summer hemisphere).

Figure 3(a-f) is the same as Fig. 2 except for the constant pressure surfaces of about 134 (a), 150 (b), 180 (c), 203 (d), 306 (e), and $501 \mathrm{~km}$ (f). The maximum wind velocity in each panel is 165 (a), 195 (b), 243 (c), 264 (d), 355 (e), and 383 (f) $\mathrm{m} / \mathrm{s}$. The day-night temperature difference becomes more remarkable with increasing height, and the wind pattern becomes more apparent from day to night at higher latitudes. The distributions of temperature and wind are almost the same between altitudes of 306 and $501 \mathrm{~km}$

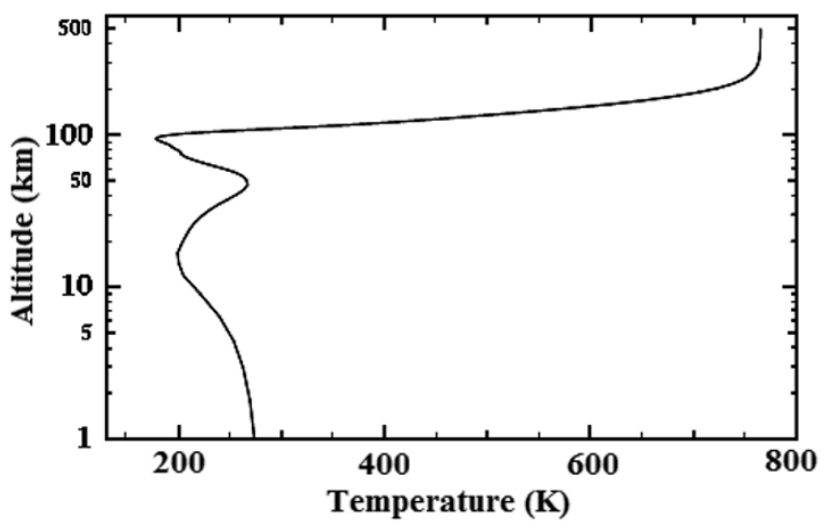

Fig. 1. Global mean temperature from near the ground surface to the exobase at 00:00 UT on November 5 calculated by a whole atmosphere general circulation model. A solar minimum and geomagnetically quiet condition is assumed. 


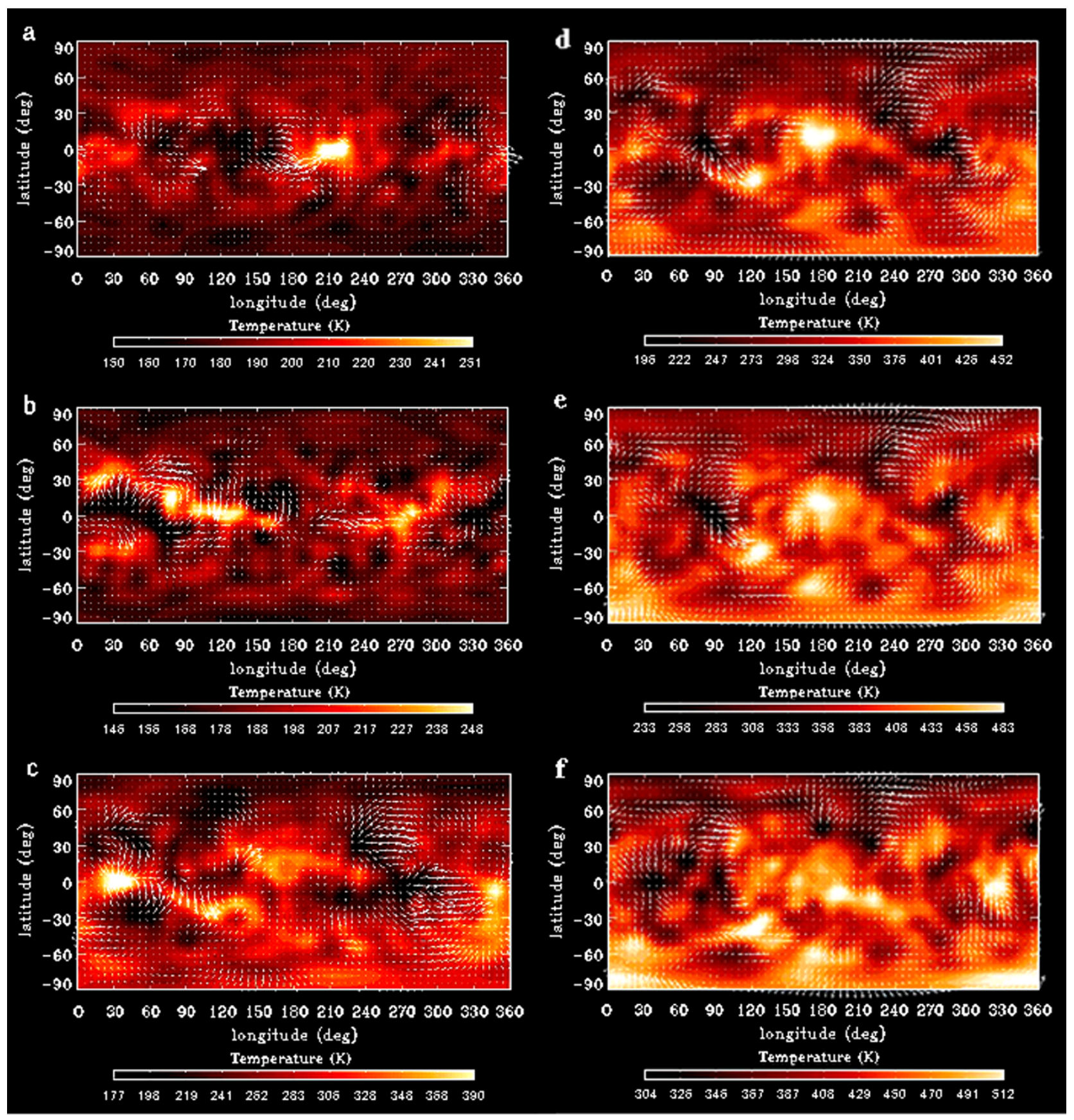

Fig. 2. Horizontal distributions of temperature and horizontal wind pattern on the constant pressure surfaces of about (a) 90 , (b) 96 , (c) 108 , (d) 112 , (e) 116, and (f) $122 \mathrm{~km}$ at 00:00 UT on November 5. Note that the temperature range is different in each panel. The maximum wind velocity in each panel is (a) 190 , (b) 182 , (c) 175 , (d) 181 , (e) 164 , and (f) $144 \mathrm{~m} / \mathrm{s}$.

because of large molecular diffusions in the upper thermosphere. Although the molecular diffusion is large at the top of the thermosphere, small-scale temperature structures still remain in the altitude region. Figure 3 also shows that the altitude regions between about 122 and $180 \mathrm{~km}$ (Figs. 2(f), $3(\mathrm{c}))$ are the transient regions from the middle to upper atmosphere because day-night variations of temperature and wind patterns change from a semi-diurnal variation to a diurnal one due to in situ forcings.

In the run where lower atmospheric effects are to be suppressed, we set the winds and temperatures below 80 $\mathrm{km}$ to zero and to horizontally uniform global mean values, respectively. Figure 4 shows the horizontal distribu- tions of temperature and horizontal wind pattern on the constant pressure surface of about $306 \mathrm{~km}$ under the conditions of a solar minimum, geomagnetically quiet, and a time of 00:00 UT on November 6. Figure 4(a, b) shows the usual calculation results (including the lower atmospheric effects) and calculation results without the lower effects, respectively. The temperature and wind shown in Fig. 4(b) are the results of a 1-day calculation after setting the global mean temperature and zero-wind below about an altitude of $80 \mathrm{~km}$. The maximum wind velocity in each panel of Fig. 4 is 350 (a) and 378 (b) m/s. It is clear that the temperature distribution shown in Fig. 4(b) is smoother than that shown in Fig. 4(a). The temperature structures extend- 


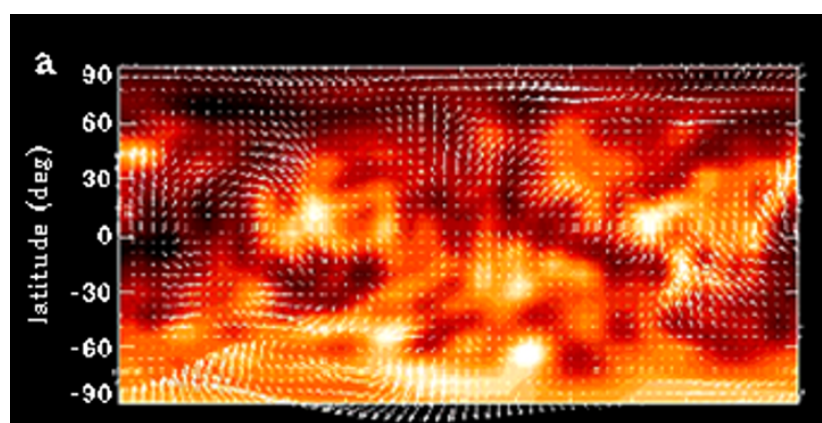

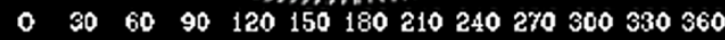
longitude (deg) Temperature (K)

$\begin{array}{lllllllllll}375 & 398 & 421 & 445 & 468 & 491 & 515 & 538 & 562 & 585 & 508\end{array}$

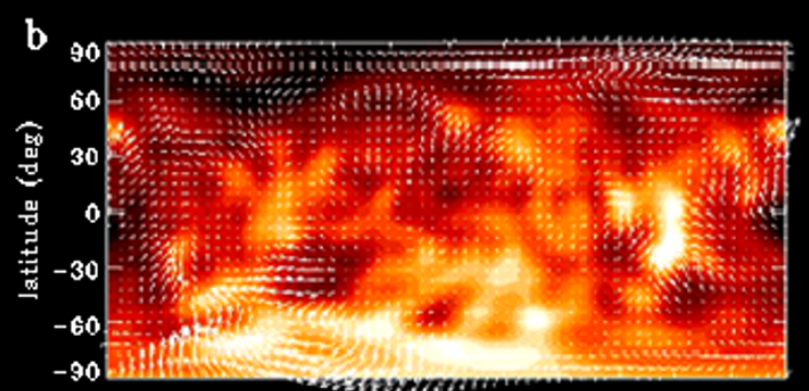

- 30 $60 \quad 90 \quad 120 \quad 150 \quad 180 \quad 210 \quad 240 \quad 270 \quad 300 \quad 330 \quad 360$ longitude (deg)

Temperature (K)
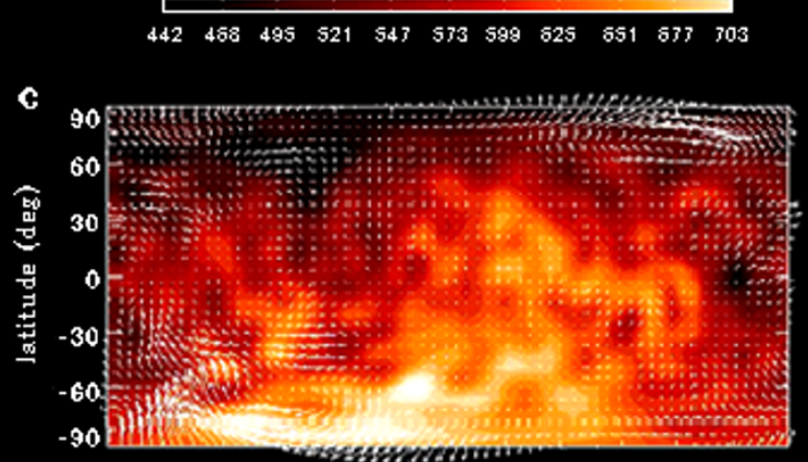

- $30 \quad 60 \quad 90 \quad 120 \quad 150 \quad 180 \quad 210 \quad 240 \quad 270 \quad 300 \quad 330 \quad 360$ longitude (deg)

Temperature (K)

$\begin{array}{lllllllllll}528 & 560 & 593 & 625 & 653 & 689 & 322 & 354 & 386 & 818 & 851\end{array}$

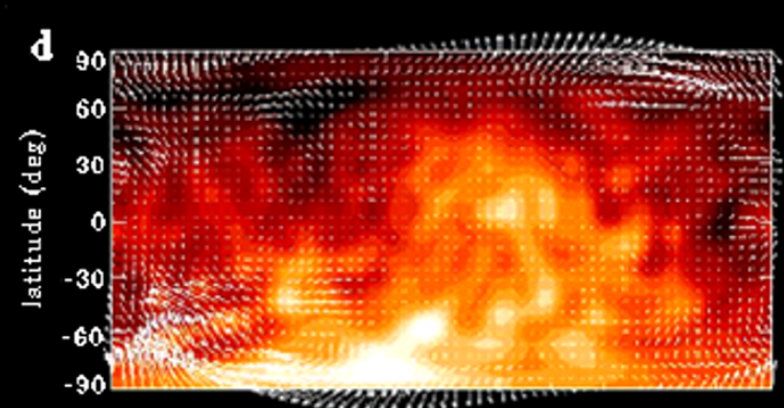

- 30 $60 \quad 90 \quad 120 \quad 150 \quad 180 \quad 210240 \quad 270 \quad 300 \quad 330 \quad 360$ longitude (deg)

Temperature (K)

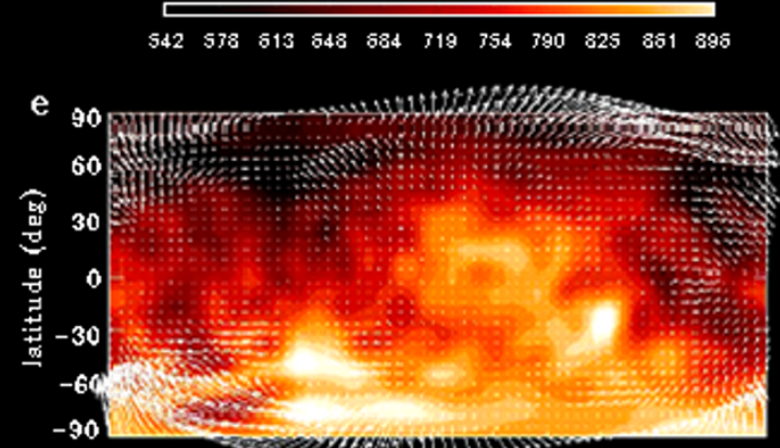

- 30 60 90 150 fed 160, 10240270300330360 longitude (des) Temperature (K)

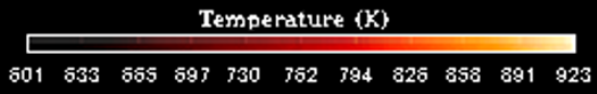

f

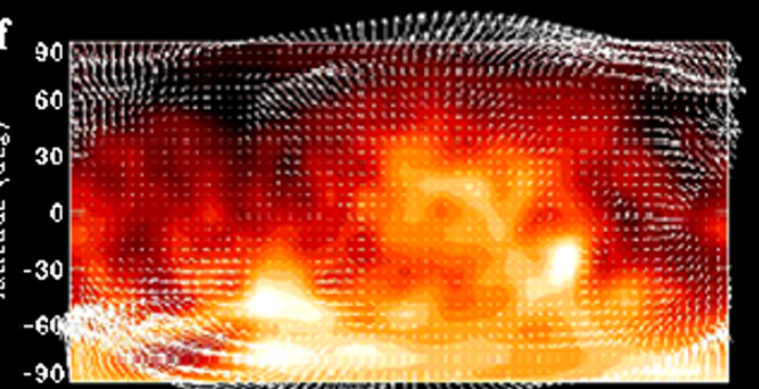

- 30 6090260760 180 210240270300330360 longitude (deg)

Temperature (K)

\begin{tabular}{lllllllllll}
\hline 08 & 639 & 670 & 301 & 732 & 362 & 393 & 824 & 855 & 886 & 913
\end{tabular}

Fig. 3. Same as Fig. 2 (temperature and wind at 00:00 UT on November 5) except for the constant pressure surfaces of about (a) 134, (b) 150, (c) 180, (d) 203 , (e) 306 , and (f) $501 \mathrm{~km}$. The maximum wind velocity in each panel is (a) 165 , (b) 195, (c) 243, (d) 264, (e) 355 , and (f) $383 \mathrm{~m} / \mathrm{s}$.

ing in the latitudinal direction at the mid- and low-latitudes are particularly remarkable in Fig. 4(a), while these structures are smoothed out in Fig. 4(b). The temperature range in Fig. 4(a) is also different from that in Fig. 4(b); namely, 607-946 K and 676-949 K, respectively, depending on the lower atmospheric variation from hour to hour.

In order to investigate thermospheric disturbances during/after passages of TADs, we performed numerical simulations for geomagnetically disturbed (during the first $1 \mathrm{~h}$, $\Phi$ is enhanced to $60 \mathrm{kV}$ and is set to $30 \mathrm{kV}$ again) periods with and without the lower atmospheric effects. Figure 5 shows the temperature and wind distributions on the constant pressure surface of about $306 \mathrm{~km}$ during periods of a 1-h enhancement of high-latitude energy inputs (Fig. 5(a, c)) and $3 \mathrm{~h}$ after the cessation of the high-latitude energy inputs (Fig. 5(b, d)), namely, at 01:00 UT and 04:00 UT on November 6 . The calculation results shown in Fig. 5(a,b) include the lower atmospheric effects, while those shown in Fig. 5(c, d) do not. Note that the range of temperature variation is different in each panel. As mentioned above, temperature at an altitude of about $306 \mathrm{~km}$ becomes slightly higher when the lower atmospheric effects are suppressed in the present case. The maximum wind velocity seen in each panel of Fig. 5 is 511 (a), 304 (b), 485 (c), and 355 (d) $\mathrm{m} / \mathrm{s}$. In Fig. 5(a, c), high-temperature regions are seen in the longitudinal direction in latitudes higher than about $60^{\circ}$. In the southern hemisphere, high-temperature regions are also seen at a latitude of about $30^{\circ}$ around a $90^{\circ}$ longitude. 


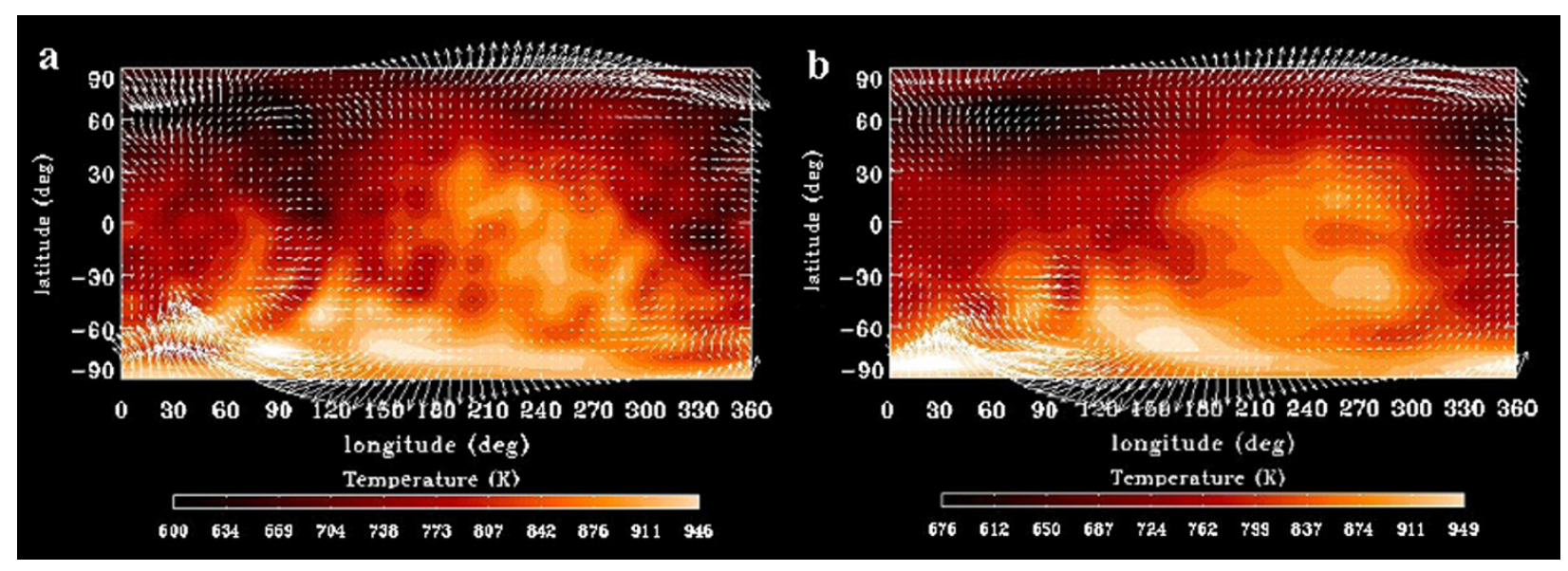

Fig. 4. Horizontal distributions of temperature and horizontal wind pattern on the constant pressure surface of about $306 \mathrm{~km}$ under the condition of solar minimum, geomagnetically quiet, and at 00:00 UT on November 6: (a) usual calculation result (including the lower atmospheric effects) , (b) calculation result without the lower effects. The maximum wind velocity in each panel is (a) 350 and (b) $378 \mathrm{~m} / \mathrm{s}$.
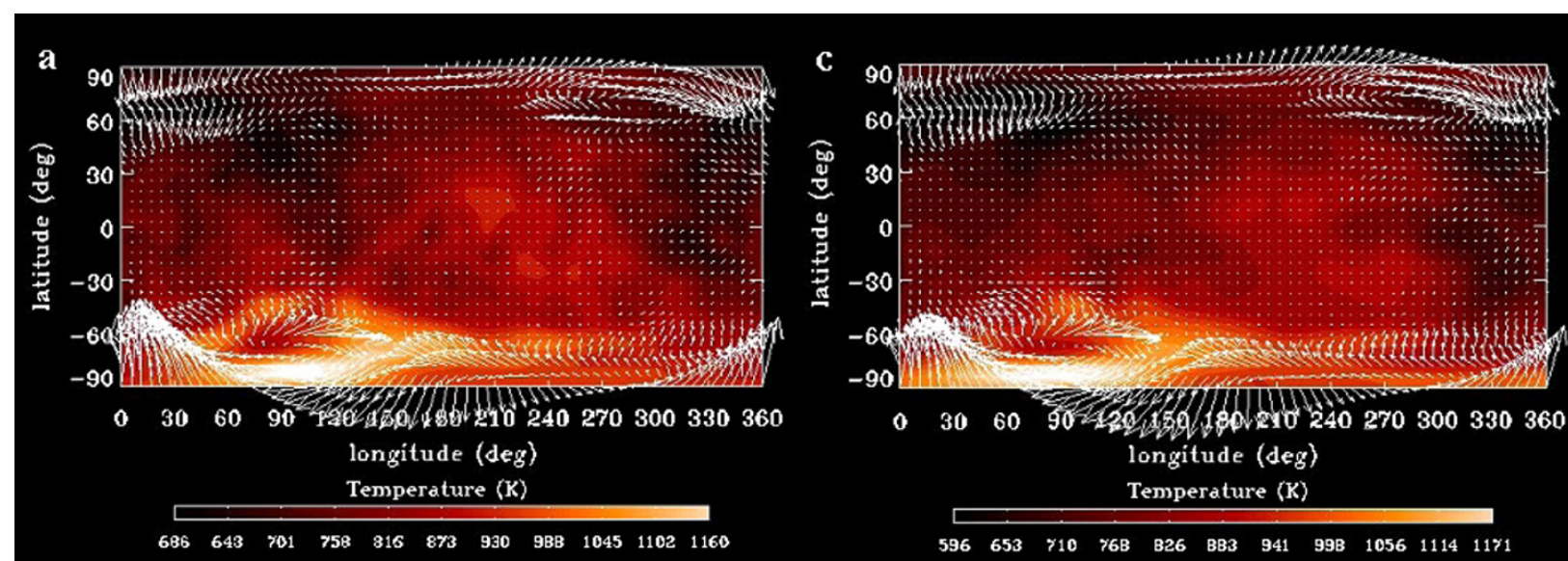

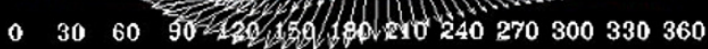
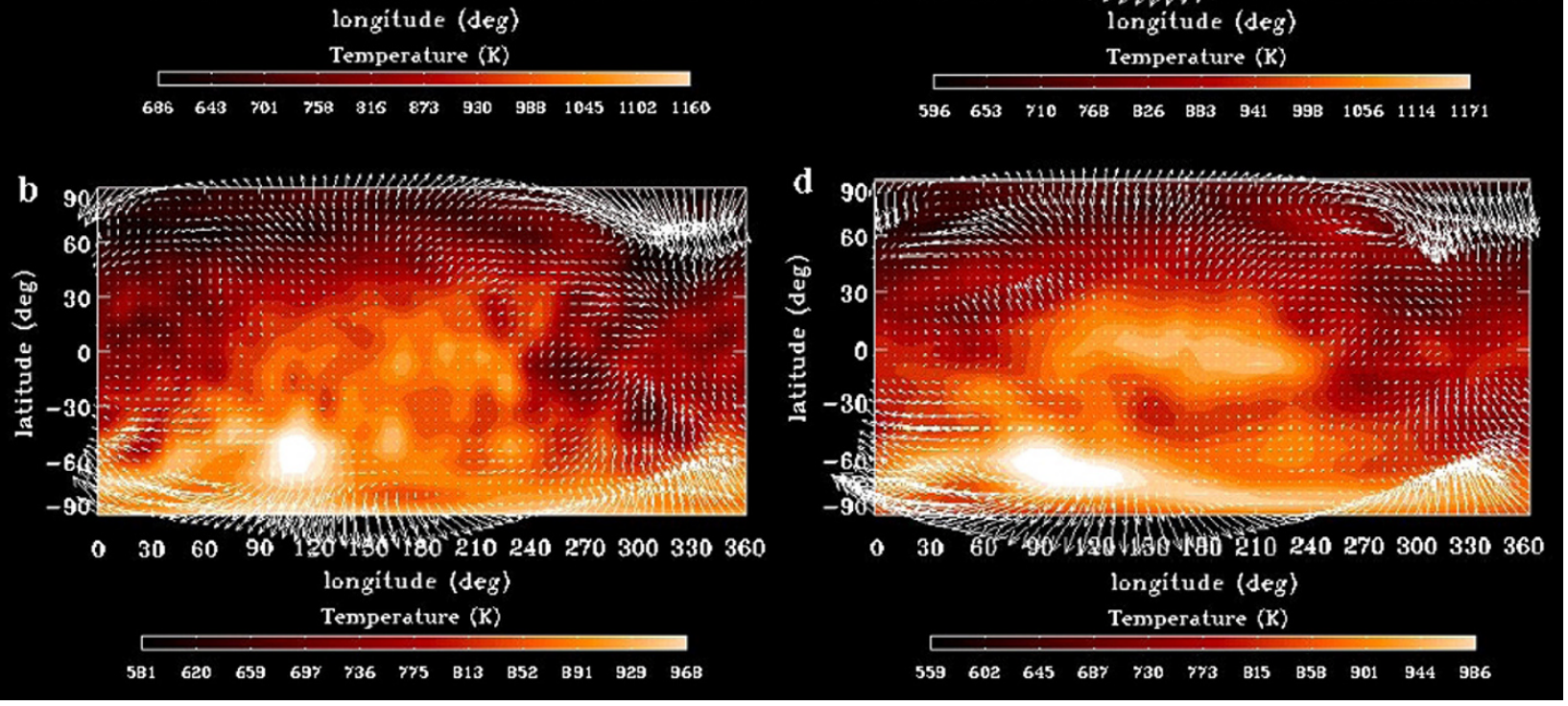

Fig. 5. Temperature and wind distributions on the constant pressure surface of about $306 \mathrm{~km}$ during periods of a 1-h enhancement of high-latitude energy inputs (a, c) and $3 \mathrm{~h}$ after the cessation of the enhanced high-latitude energy inputs (b, d): namely, at 01:00 UT and 04:00 UT on November 6, respectively. The results shown in the left panels (a, b) include the lower atmospheric effects, while those shown in the right panels (c, d) do not. The maximum wind velocity in each panel is (a) 511 , (b) 304 , (c) 485 , and (d) $355 \mathrm{~m} / \mathrm{s}$.

The longitudinal extents of the high-temperature regions are manifestations of the fronts of the large-scale TADs. Both the equator- and pole-ward propagating TADs appear in the high-latitude regions. Strong winds in the high-latitude region seem to be driven because the superposition of the TAD-induced and localized temperature structures produce large pressure gradients in Fig. 5(a). As seen in Fig. 5(b, d), high-temperature regions appear in the low-latitude regions due to TAD propagations from both hemispheres. The TAD fronts are clearly seen in Fig. 5(d) (case of suppressing the lower atmospheric effects), while longitudinal temperature structures extending in the north-south direction are superimposed on TADs in Fig. 5(b), particularly in the midand low-latitude regions. In addition, distinct TAD fronts 


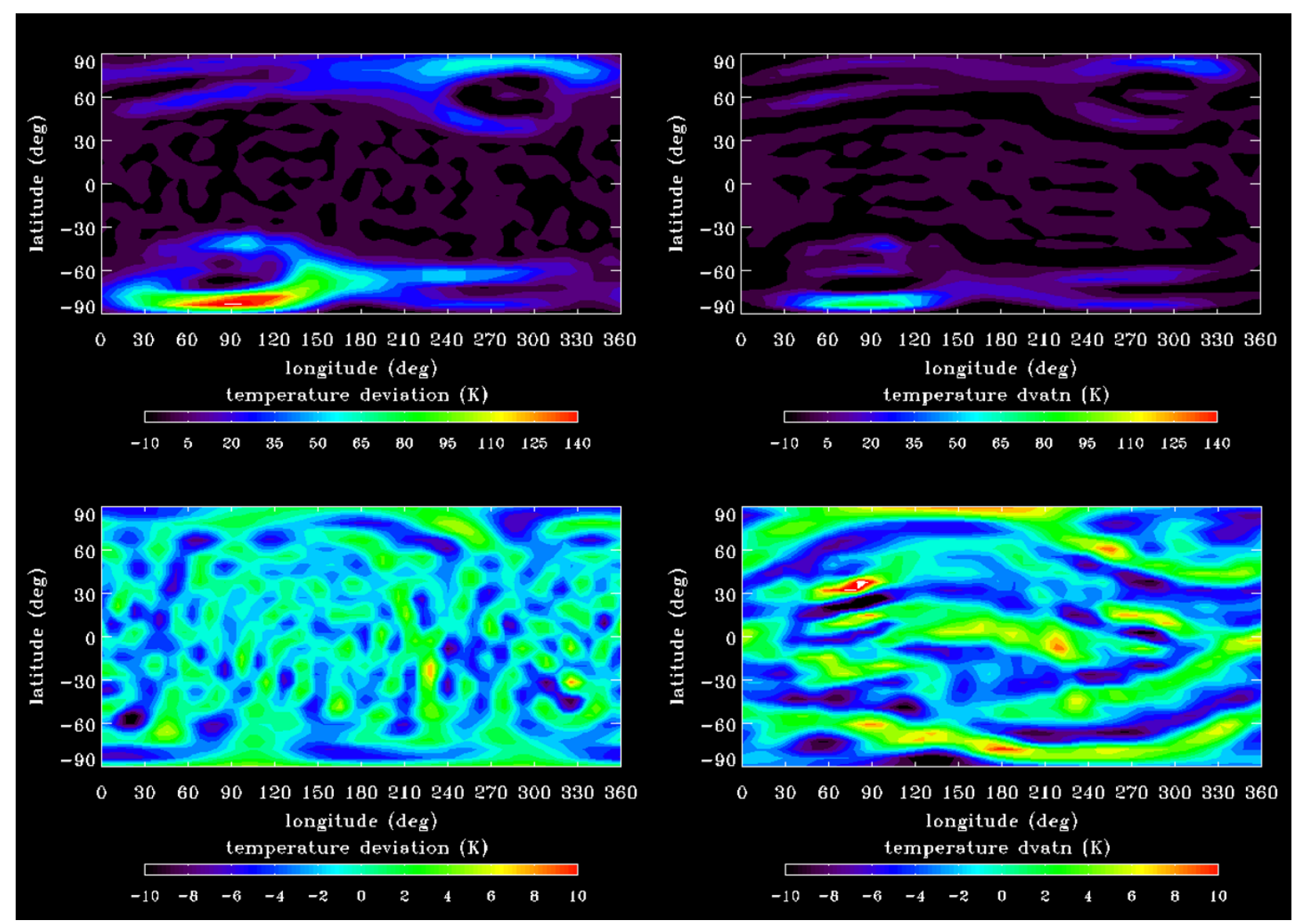

Fig. 6. Same as Fig. 5 except for the temperature differences between the temperatures at UT = 01:00, 04:00 and the 40-min (UT = 00:40-01:20, 03:40-04:20) averaged ones. Figure 6(a) and (b) are those in the case with the lower atmospheric effects. Figures 6(c) and (d) are those in the case without the lower atmospheric effects.

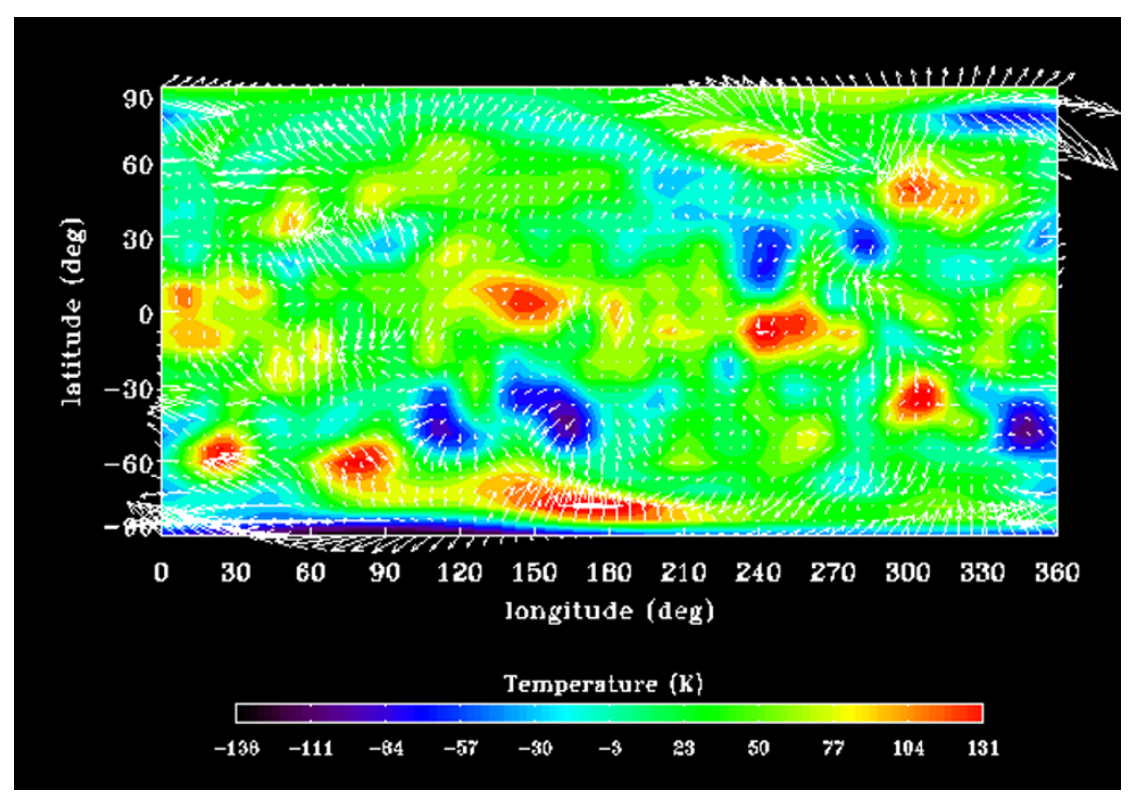

Fig. 7. Differences in temperature and wind at 04:00 UT on November 6 between those cases shown in Fig. 5(d) and (b). The temperature difference is in the range between -138 and $131 \mathrm{~K}$. The maximum difference-wind velocity is $169 \mathrm{~m} / \mathrm{s}$.

seen in Fig. 5(d) produce large pressure gradients that drive winds that are stronger than those seen in Fig. 5(b), in wideranging areas (e.g., winds in the northern high latitudes at about $30^{\circ}-180^{\circ}$ longitude and mid-latitudes at about $30^{\circ}-$ $90^{\circ}$ longitude).

In order to draw out TAD and disturbed components of temperature, the differences between the temperatures at $\mathrm{UT}=01: 00$ and 04:00 and the 40-min (UT $=00: 40-01: 20$, 
03:40-04:20) averaged ones are shown in Fig. 6. The temperature differences in cases with and without the lower atmospheric effects are shown in Fig. 6(a, b) and 6(c, d) respectively. In the present case, the amplitudes of TADs generated in Fig. 6(a) are larger than those generated in Fig. 6(c). However, the amplitudes of TADs propagating from the high- to low-latitude regions are larger in Fig. 6(d) than in Fig. 6(b). The TAD fronts extending in the longitudinal direction are clearly seen in Fig. 6(d), while more localized structures extending in the latitudinal direction (spatial extent of about $15^{\circ}$ ) are seen in Fig. 6(b), particularly around the solar terminator (the region between $210^{\circ}-240^{\circ}$ longitude).

Figure 7 shows the differences in temperature and wind between those shown in Fig. 5(b) and (d). The maximum difference-wind in Fig. 7 is $169 \mathrm{~m} / \mathrm{s}$. Although the ranges of temperature in Fig. 5(b) and 5(d) are almost the same, the range of temperature difference shown in Fig. 7 is between -138 and $131 \mathrm{~K}$. This means that the lower atmospheric effects can contribute to determining the locations of high- and low-temperature regions during/after passages of TADs. The disturbances in temperature with longitudinal scales of about $15^{\circ}-30^{\circ}$ are notable in the low-latitude region. The difference-winds blow in the direction of TAD propagation almost along the TAD fronts, suggesting abatements of TAD-induced winds by the lower atmospheric effects.

\section{Discussion}

We have obtained an overview of the morphological characteristics of the thermospheric disturbances globally produced by the effects from both the lower atmosphere and the energy inputs from the magnetosphere into the polar thermosphere. At earlier stages of the thermospheric GCM simulation, the models calculated a largescale smoothly varying temperature and wind field because of the fixed lower boundary at around $80-100 \mathrm{~km}$. We can now show the lower atmospheric effects on various structures/disturbances in the thermosphere in our GCM results.

Since the lower atmospheric effects seem to result from momentum and energy transfer mainly due to atmospheric wave propagation, dissipation, breakdown, and secondary waves from break-down waves, it is quite difficult to understand the mechanisms for producing individual thermospheric disturbances. In the GCM simulations, parameterizations are used to represent the atmospheric wave effects. Our first step for investigating the lower atmospheric effects on the upper atmosphere was to compare the usual GCM simulations with simulations using the global mean temperature and zero-wind below an altitude of about $80 \mathrm{~km}$. In order to understand the coupling mechanisms between the lower and upper atmospheres, we need to investigate the characteristics of atmospheric waves as presented by Miyoshi and Fujiwara (2006, 2008, 2009).

Some previous observations have shown localized structures or day-to-day variability in temperature, wind, composition, and mass density in the upper (or $F$-region) thermosphere (Colerico et al., 1996; Aruliah and Griffin, 2001; Strickland et al., 2001; Bruinsma et al., 2006; Bruinsma and Forbes, 2007). Colerico et al. (1996) observed significant day-to-day variability in the midnight temperature maximum (MTM) whose midnight pressure bulge leads to neutral wind variations. Aruliah and Griffin (2001) observed significant meso-scale winds in addition to a smoothly varying large-scale wind field in the high-latitude region. The GCM simulations presented here also showed localized temperature and wind structures that vary from hour to hour. The day-to-day variability in the thermosphere and ionosphere previously observed may depend on that of the localized structures which appeared in our study. As seen in Figs. 5 and 7, the temperature structures (or pressure bulges) seem to abate TAD-induced winds, suggesting the importance of understanding the significance of localized thermospheric structures/disturbances and MTM in order to comprehend thermospheric/ionospheric dynamics.

As seen in Fig. 6, the localized thermospheric structures are important for TAD generation and propagation; namely, the lower atmospheric effects contribute to the enhancement of the TAD amplitudes generated in the high-latitude region, while the lower atmospheric effects abate the TAD amplitudes propagating from the high- to low-latitude regions in the present case. Since GCM simulations show day-to-day variations in the localized thermospheric structures originating from the lower atmospheric effects (the results are not shown here), the TAD amplitude and/or its propagated distance should be variable from day to day depending on season, solar cycle, and geomagnetic activity. Our results suggest that the lower atmospheric effects are an important cause of the variability of TADs.

In our study, we observed localized thermospheric structures/disturbances, particularly in the equatorial region. We will investigate more details of the structures/disturbances, such as seasonal and solar cycle dependence, in the near future. Recent observations suggest a strong coupling between the equatorial troposphere and ionosphere through propagations of the atmospheric gravity waves (e.g., Ogawa et al., 2006). Nicolls and Kelley (2005) also showed an observational evidence for the gravity wave seeding of ionospheric plasma instability. These researchers suggested that the large-scale traveling ionospheric disturbance (LSTID) created conditions conducive to instability, while the shorter period waves created the seed irregularities. Our simulations also suggest that interaction between the lower atmospheric effects and TAD will contribute to create conditions conducive to instability. A self-consistent modeling of the atmosphere and ionosphere, which is our current project, will enable us to investigate the above problem.

The relationship between gravity wave activity in the equatorial region, resultant thermospheric dynamics, and ionospheric phenomena, such as plasma bubbles and equatorial spread $F$ (ESF), should be investigated in both observational and theoretical studies. The equatorial radar facility (Fukao, 2007) and future satellites, namely, the Ionosphere, Mesosphere, upper Atmosphere, and Plasmasphere mapping satellite (IMAP satellite), will provide us with important information on coupling between the ionosphere and atmosphere. 


\section{Summary}

The GCM simulations presented here, which include lower atmospheric effects on the thermosphere, show that localized structures/disturbances appear globally in the upper (or $F$-region) thermosphere. Below an altitude of about $120-130 \mathrm{~km}$, the temperature and wind are largely controlled by the lower atmospheric effects, while day-night variation and the effects of the high-latitude energy inputs and ion-drag become larger with increasing height. The thermospheric localized structures are not as remarkable in the upper altitude region as in the lower altitude region because of large molecular diffusion/viscosity.

Based on the numerical simulations with and without the lower atmospheric effects, we found that the localized longitudinal structures/disturbances extending in the latitudinal direction are produced by the lower atmospheric effects. The disturbances with longitudinal scales of about $15^{\circ}-30^{\circ}$ are notable in our study. In the case without the lower atmospheric effects, more smoothly varying temperature and wind structures are set up in the thermosphere.

The localized structures/disturbances in the thermosphere sometimes result from a superposition of the lower atmospheric effects and TADs generated due to the high-latitude energy inputs. The thermospheric structures/disturbances produced by the lower atmospheric effects abate the TAD-induced winds, suggesting the importance of localized thermospheric structures/disturbances in thermospheric/ionospheric dynamics, particularly in the mid- and low-latitude regions.

The localized temperature and wind structures shown in our GCM simulations vary from hour to hour. The day-to-day variability of the thermosphere and ionosphere previously observed would depend on variations in such structures/disturbances. Our results suggest that variable thermospheric structures/disturbances originating from the lower atmosphere can contribute to producing variability in TADs and plasma instability.

Acknowledgments. This work was supported in part by Grant-inAid for Scientific Research C (20540435), B (17340140), and the $21^{\text {st }}$ Century COE program "Advanced Science and Technology Center for the Dynamic Earth" by the Ministry of Education, Science, Sports and Culture, Japan, and the joint research program of the Solar-Terrestrial Environment Laboratory, Nagoya University.

\section{References}

Aruliah, A. L. and E. Griffin, Evidence of meso-scale structure in the highlatitude thermosphere, Ann. Geophys., 19, 37-46, 2001.

Balan, N., S. Kawamura, T. Nakamura, M. Yamamoto, S. Fukao, W. L. Oliver, M. E. Hagan, A. D. Aylward, and H. Alleyne, Simultaneous mesosphere-lower thermosphere and thermospheric $F$ region observations using middle and upper atmosphere radar, J. Geophys. Res., 111, A10S17, doi:10.1029/2005JA011487, 2006.

Balthazor, R. L. and R. J. Moffett, Morphology of large-scale traveling atmospheric disturbances in the polar thermosphere, J. Geophys. Res., 104, 15-24, 1999.

Bruinsma, S. and J. M. Forbes, Global observation of traveling atmospheric disturbances (TADs) in the thermosphere, Geophys. Res. Lett., 34, L14103, doi:10.1029/2007GL030243, 2007.

Bruinsma, S., J. M. Forbes, R. S. Nerem, and X. Zhang, Thermosphere density response to the 20-21 November 2003 solar and geomagnetic storm from CHAMP and GRACE accelerometer data, J. Geophys. Res., 111, A06303, doi:10.1029/2005JA011284, 2006.
Chiu, Y. T., An improved phenomenological model of ionospheric density, J. Atmos. Terr. Phys., 37, 1563-1570, 1975.

Colerico, M., M. Mendillo, D. Nottingham, J. Baumgardner, J. Meriwether, J. Mirick, B. W. Reinisch, J. L. Scali, C. G. Fesen, and M. A. Biondi, Coordinated measurements of $F$ region dynamics related to the thermospheric midnight temperature maximum, J. Geophys. Res., 101, 26,783-26,793, 1996.

Fujiwara, H. and Y. Miyoshi, Characteristics of the large-scale traveling atmospheric disturbances during geomagnetically quiet and disturbed periods simulated by a whole atmosphere general circulation model, Geophys. Res. Lett., 33, L20108, doi:10.1029/2006GL027103, 2006.

Fujiwara, H., S. Maeda, H. Fukunishi, T. J. Fuller-Rowell, and D. S. Evans, Global variations of thermospheric winds and temperatures caused by substorm energy injection, J. Geophys. Res., 101, 225-239, 1996.

Fukao, S., Recent advances in atmospheric radar study, J. Meteor. Soc. Jpn., 85B, 215-239, 2007.

Fuller-Rowell, T. J. and D. S. Evans, Height-integrated Pedersen and Hall conductivity patterns inferred from the TIROS-NOAA satellite data, $J$. Geophys. Res., 92, 7606-7618, 1987.

Immel, T. J., E. Sagawa, S. L. England, S. B. Henderson, M. E. Hagan, S. B. Mende, H. U. Frey, C. M. Swenson, and L. J. Paxton, Control of equatorial ionospheric morphology by atmospheric tides, Geophys. Res. Lett., 33, L15108, doi:10.1029/2006GL02616, 2006.

Lühr, H., K. Häusler, and C. Stolle, Longitudinal variation of $F$ region electron density and thermospheric zonal wind caused by atmospheric tides, Geophys. Res. Lett., 34, L16102, doi:10.1029/2007GL030639, 2007.

Mendillo, M., H. Rishbeth, R. G. Roble, and J. Wroten, Modelling F2layer seasonal trends and day-to-day variability driven by coupling with the lower atmosphere, J. Atmos. Sol.-Terr. Phys., 64, 1911-1931, 2002.

Millward, G. H., R. J. Moffett, and S. Quegan, Effects of an atmospheric gravity wave on the midlatitude ionospheric $F$ layer, J. Geophys. Res., 98, 19,173-19,179, 1993.

Miyahara, S., Y. Yoshida, and Y. Miyoshi, Dynamic coupling between the lower and upper atmosphere by tides and gravity waves, J. Atmos. Terr. Phys., 55, 1039-1053, 1993.

Miyoshi, Y., Numerical simulation of the 5-day and 16-day waves in the mesopause region, Earth Planets Space, 51, 763-772, 1999.

Miyoshi, Y. and H. Fujiwara, Day-to-day variations of migrating diurnal tide simulated by a GCM from the ground surface to the exobase, Geophys. Res. Lett., 30, 1789, doi:10.1029/2003GL017695, 2003.

Miyoshi, Y. and H. Fujiwara, Excitation mechanism of intraseasonal oscillation in the equatorial mesosphere and lower thermosphere, J. Geophys. Res., 111, D14108, doi:10.1029/2005JD006993, 2006.

Miyoshi, Y. and H. Fujiwara, Gravity waves in the thermosphere simulated by a general circulation model, J. Geophys. Res., 113, doi:10. 1029/2007JD008874, 2008.

Miyoshi, Y. and H. Fujiwara, Gravity waves in the equatorial thermosphere and their relation to lower atmospheric variability, Earth Planets Space, 61, this issue, 471-478, 2009.

Nicolls, M. J. and M. C. Kelley, Strong evidence for gravity wave seeding of an ionospheric plasma instability, Geophys. Res. Lett., 32, L05108, doi:10.1029/2004GL020737, 2005.

Ogawa, T., Y. Otsuka, K. Shiokawa, A. Saito, and M. Nishioka, Ionospheric disturbances over Indonesia and their possible association with atmospheric gravity waves from the troposphere, J. Meteor. Soc. Jpn., CPEA Special Issue, 84A, 327-342, 2006.

Roble, R. G. and E. C. Ridley, An auroral model for the NCAR thermospheric general circulation model (TGCM), Ann. Geophys., 54, 369382, 1987.

Strickland, D. J., J. D. Craven, and R. E. Daniell, Jr., Six days thermospheric- ionospheric weather over the Northern Hemisphere in late September 1981, J. Geophys. Res., 106, 30,291-30,306, 2001.

Takahashi, H., L. M. Lima, C. M. Wrasse, M. A. Abdu, I. S. Batista, D. Gobbi, R. A. Buriti, and P. P. Batista, Evidence on 2-4 day oscillations of the equatorial ionosphere h'F and mesospheric airglow emissions, Geophys. Res. Lett., 32, L12102, doi:10.1029/2004GL022318, 2005.

Volland, H., Models of the global electric fields within the magnetosphere, Ann. Geophys., 31, 159-173, 1975.

H. Fujiwara (e-mail: fujiwara@pat.geophys.tohoku.ac.jp) and Y. Miyoshi 